\title{
Flow diagnostics using fibre optics
}

\author{
B VASUDEVAN, SRIKANTH PADBIDRI and \\ M CHANDRA KISHORE
}

\author{
Department of Aerospace Engineering, Indian Institute of Science (IISc), \\ Bangalore 560012 \\ e-mail: \{vasu,padbidri@aero.iisc.ernet.in\}
}

\begin{abstract}
Research in the area of flow diagnostics using fibre-optics started in our laboratory in early 1998 . The first-ever multi-component wind tunnel balance in the world, working with fibre-optic sensors was built and demonstrated in 1999. Since then, several new applications of the technique in the area of fluid dynamic load measurements have been tried successfully. Very recently, fibre-optic sensors have been effectively used for underwater applications, where conventional measurements are relatively very difficult. Since, different physical perturbations affect optical power flowing in a fibre in different ways, unique signatures can be obtained which allow absolute or relative measurement of the incident disturbances. Immunity to electromagnetic or radio frequency interference, high temperature capability, low fatigue, high sensitivity, small size, good corrosion resistance and the capability to embed sensors within the model surface are some of the very attractive features of fibre-optic based instrumentation systems. In this paper, we describe the results of experiments of aerodynamic load measurements at hypersonic speeds (Mach 8.35 and 7.0) and studies carried out recently in a water tunnel over a lifting hypersonic vehicle with a 2-component fibre-optic strain-gauge balance.
\end{abstract}

Keywords. Fibre optics; flow diagnostics; hypersonic speeds.

\section{Introduction}

Over the years, conventional technology has evolved to a fairly high level, permitting measurements of stress/strain, force, pressure, temperature, displacement, acceleration etc. with very good accuracy. However, in unfavorable conditions of fluid flow, measurements tend to be difficult, error-prone and sometimes almost impossible to make. For example, it is very difficult to measure stress/strain levels in load cells or force balances using the conventional foil type resistive elements (strain gauges) in situations where the surface undergoing strain is heated beyond a few hundred degrees. Even at $300^{\circ} \mathrm{C}$, conventional strain measurement suffers a fall in accuracy to uncomfortable levels (more than 5\%) and the measurement becomes meaningless at $500^{\circ} \mathrm{C}$ and above. A technology has been slowly evolving over the last decade which gives a distinct advantage under these conditions of flow. We refer here to the rapid strides made in the development of fibre-optic sensors and associated signal 


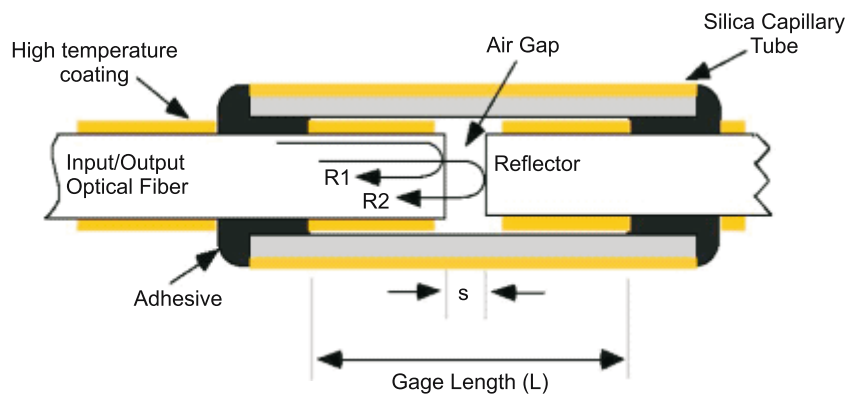

Figure 1. Close-up view of EFPI fibre-optic strain gauge.

processors. The beauty of the fibre-optic sensor, for example, is in the ability to measure strain on a heated surface in a combustion chamber where the temperature could be as high as $1900^{\circ}$ C. Sapphire wave guides carrying an optical beam generated by low power solidstate lasers permit such strain measurements. Additionally, these fibre-optic strain gauges are extremely compact, an order of magnitude smaller than the smallest resistive strain gauge ever made.

\section{Six-component internal fibre-optic strain gauge balance}

Figure 1 shows a close-up view of EFPI (Extrinsic Fabry-Perot Interferometer) fibre-optic strain gauge. There are two optical paths R1 and R2 in the optical fibre which cause a phase shift in the laser light passing along them. This shift is proportional to ' $S$ ', the air gap. As the gauge undergoes strain, the air gap ' $S$ ' changes, resulting in a change in the phase shift. These gauges can pick up strain as high as $5000 \mu \in$ leading to a very large phase shift. The shifts are detected using a signal processor, which detects extremely small shifts leading to sub-micro-strain resolutions. Figure 2 presents a view of the six-component internal strain gauge balance which has been gauged with both normal foil-type resistive strain gauges, as well as fibre-optic based EFPI gauges. It is interesting to note that these fibre gauges are almost an order of magnitude smaller (sometimes less than a hundred microns in diameter) as compared to the smallest of the resistive-type strain gauges (a few millimetres long or wide). Additionally, the way the fibre-optic stain gauge works, strain sensitivity for transverse

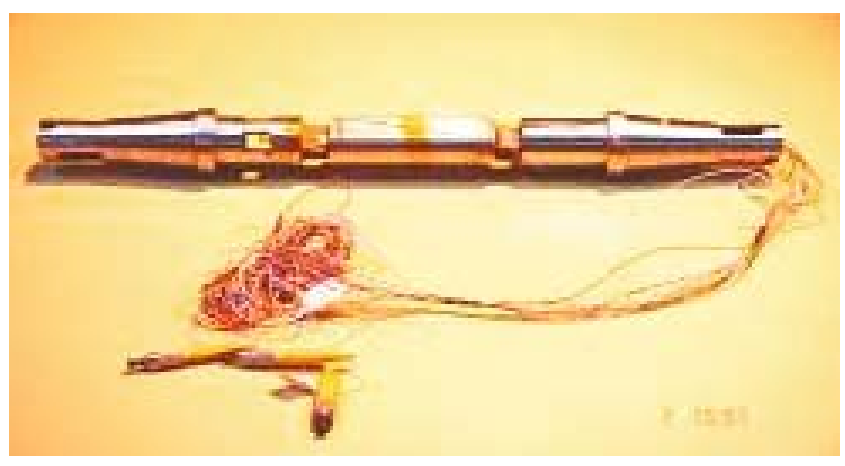

Figure 2. A view of the 6component strain-gauge balance with both EFPI fibre-optic gauges and conventional strain gauges. 


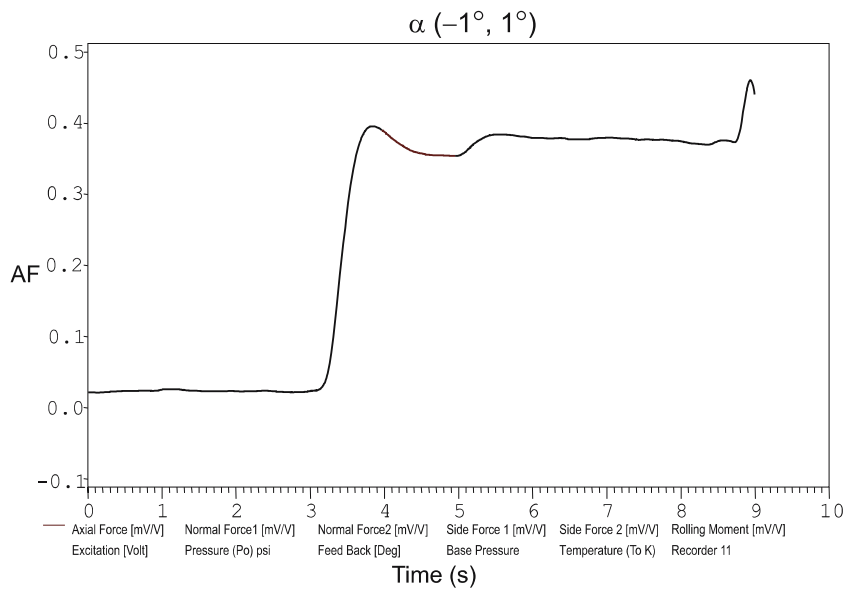

Figure 3. Performance of the fibre-optic balance in a wind tunnel.

loads is almost zero, whereas in conventional gauges, which work on the basis of geometry (length and cross-sectional area), the transverse sensitivity is quite high (a function of the Poisson ratio). Fibrescan 2000 signal processor was used for measuring the strain levels using fibre-optic stain gauges. The signal output from the axial force in a typical wind tunnel run is given in figure 3 (Vasudevan 1993). Loads measured with the conventional strain gauges work well only within a small range of operating temperature, generally less than $100^{\circ} \mathrm{C}$. However, special fibre-optic strain gauges show remarkable insensitivity to even very high temperatures (as high as $1000^{\circ} \mathrm{C}$ ) and therefore are very useful for measurement of loads in high enthalpy, high speed flows and in combustion chambers, rocket exhausts etc.

\section{Aerodynamic load measurements at hypersonic speeds using internally mounted fibre-optic balance system}

\subsection{The IISc hypersonic wind tunnel}

The IISc hypersonic wind tunnel is of the pressure-vacuum type with an open jet test section of $300 \mathrm{~mm}$ diameter, enclosed in a plenum chamber (Vasudevan 1998). There are two pressure vessels, each of $10.6 \mathrm{~m}^{3}$ capacity, which can be charged up to a pressure of $140 \mathrm{~kg} . \mathrm{f} / \mathrm{cm}^{2}$, using two $125 \mathrm{hp}$, five-stage, water-cooled, reciprocating-type compressors, each having a discharge capacity of $7 \mathrm{~m}^{3}$ of free air per minute. There are four vacuum tanks, each of $35.4 \mathrm{~m}^{3}$ capacity, which can be evacuated to a level of 1 Torr $(0.1 \mathrm{kPa})$ with a series of roots and radial piston pumps. A pebble bed heater containing $250 \mathrm{~kg}$ of densely packed alumina pebbles, which can be preheated up to a temperature of $800 \mathrm{~K}$, using a $500 \mathrm{~kW}$ liquid petroleum gas-based heating system is used in the circuit to prevent liquefaction of air at test Mach numbers ranging from $5-10$. This heater is designed to withstand a pressure of $70 \mathrm{~kg} / \mathrm{cm}^{2}$ at a temperature of $800 \mathrm{~K}$. The nozzles used are made of 17-4-PH alloy steel and the throat section has been specially designed with a thin wall so that the thermal stresses are kept within allowable limits even without any forced cooling.

\subsection{Model details}

The "Hypersonic Research Vehicle" is a technology demonstrator slated to fly at high altitudes at Mach 7.0. It was possible to test a 1:25 scale model of this vehicle at the IISc hypersonic 


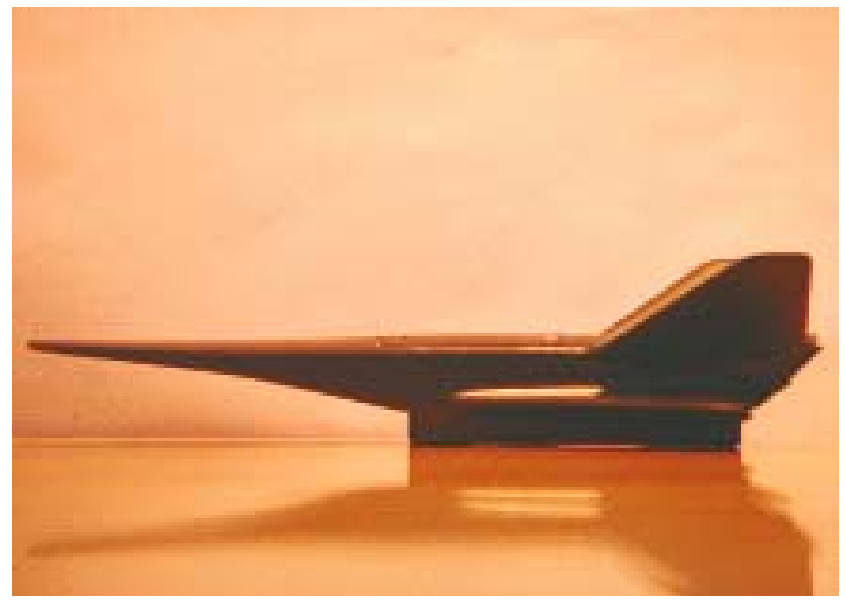

Figure 4. A photograph of the hypersonic research vehicle model used in the present studies.

wind tunnel. Figure 4 depicts the photograph of the typical scaled model of the hypersonic research vehicle. The model is made of carbon fibre with associated resins and can withstand high pressures (20 bar, $2 \mathrm{MPa}$ ) and temperatures $(800 \mathrm{~K})$. Several models with basic body and several combinations such as wings, vertical tail and flow through engine block were tested at Mach 7.0. The model is mounted in a servo-hydraulically operated sector-based model injection-cum-incidence system, which enables injection and retraction through a computeroperated control system.

\subsection{Details of the instrumentation}

The aerodynamic loads (axial force and normal forces in the present case) were measured using a conventional strain-gauge balance, shown in figure 5. The M\&M type of straingauge balance was linked to M\&M signal conditioners in conjunction with a 16-bit digital data acquisition system to obtain the aerodynamic loads. Additionally, a Lunavision-make

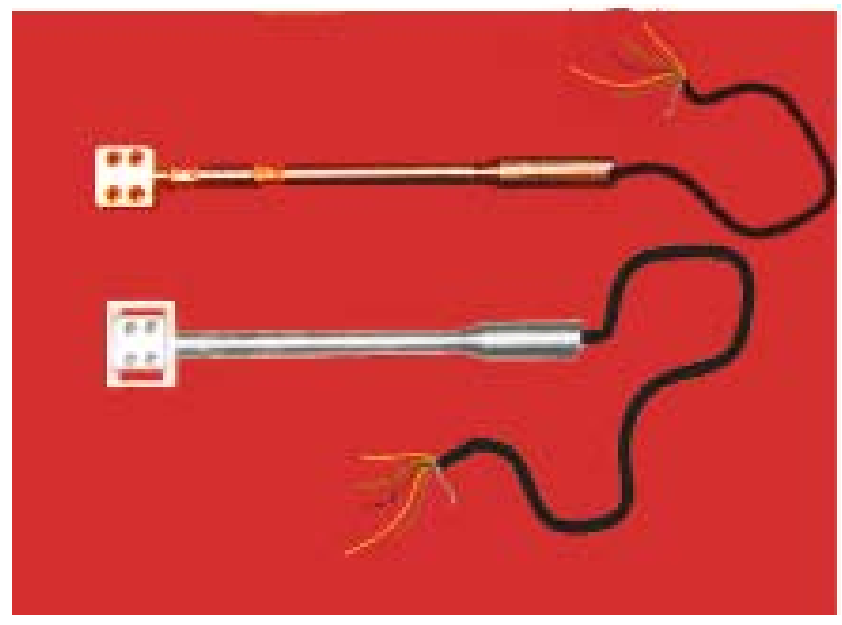

Figure 5. A photograph of the internally mountable aerodynamic force balance used in the present experiments. 


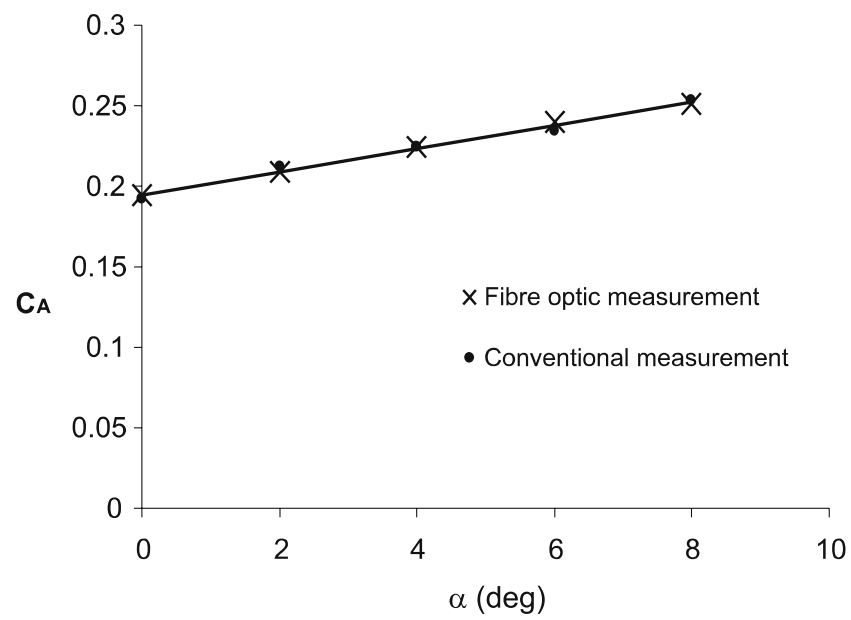

Figure 6. Variation of the drag force of the research vehicle at Mach 7 as a function of angle of incidence.

EFPI fibre-optic strain gauge was mounted onto one of the 4 axial flexures and connected to the Fibre scan 2000 fibre-optic signal processing system (Vasudevan et al 2005). Thus, a small strain which can be measured with great difficulty by conventional strain gauges can be easily measured using EFPI fibre-optic strain gauges. Fibre scan 2000 provides the necessary solid-state laser source and further processes the returning signals to provide an effective voltage output as a function of the applied axial load on the model. This processor gain can be adjusted so as to provide a $5 \mathrm{~V}$ max output for any range of strain produced in the axial flexure. In the present case, this gain was adjusted to give a maximum o/p at a strain of $150 \mu \in$ corresponding to an input drag load of $1500 \mathrm{gms}$.

\subsection{Results and discussions}

Figures 6 and 7 show charts giving the drag co-efficient and the normal force co-efficient as a function of angle of incidence $(\alpha)$. It is found that the axial flow co-efficient has been picked up by both the conventional strain-gauge system as well as the fibre-optic system. The results seem to be more or less the same indicating that the fibre-optic system is at least as good as the conventional strain-gauge system. However, on closer scrutiny, it is found that the scatter

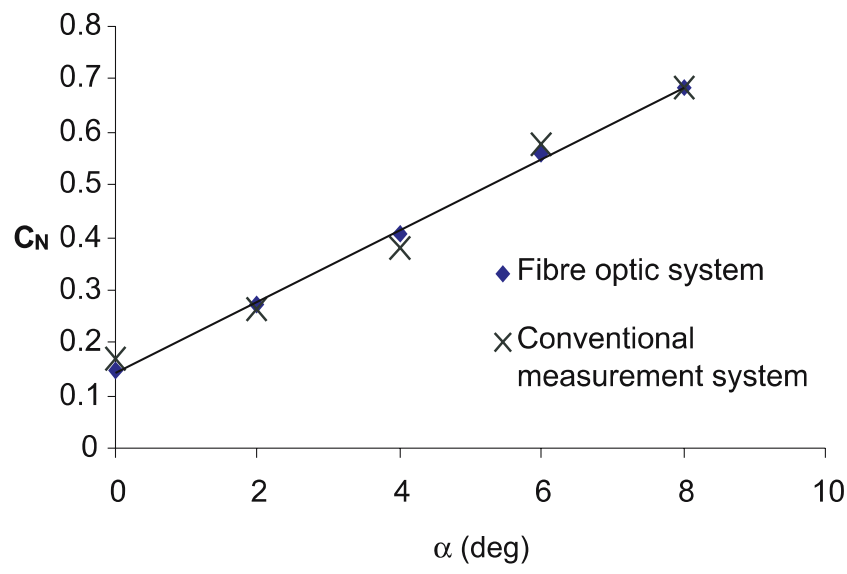

Figure 7. Variation of the normal force of the research vehicle at Mach 7 as a function of angle of incidence. 


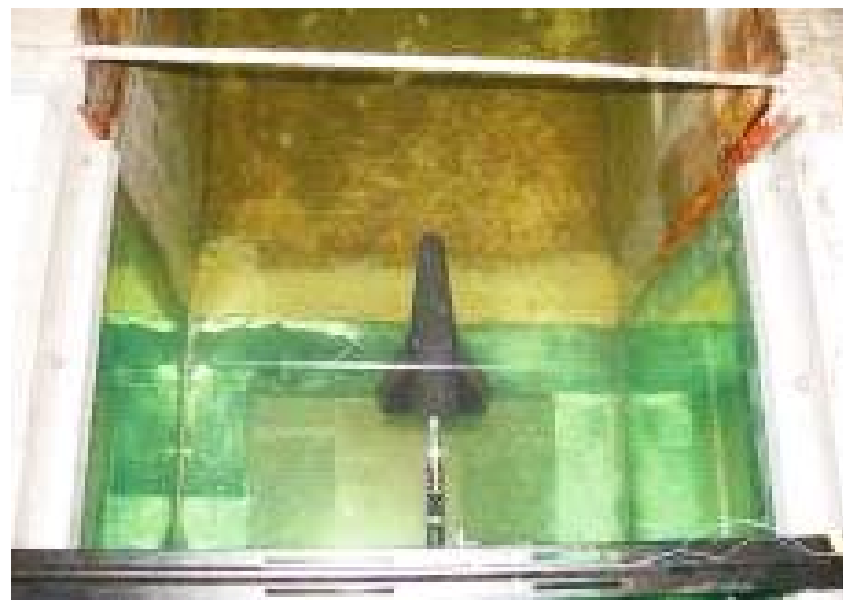

Figure 8. Variation of the normal force of the research vehicle at Mach 7 as a function of angle of incidence.

in data is much less in the fibre-optic system indicating that it is a better and more accurate instrument system. Especially at elevated temperatures, the scatter in the conventional system is likely to go up while that of the fibre-optic system is likely to be independent of the model temperatures. Also, it is practically found that the conventional measurement system picks up RFI and EMI signals also unless great care is exercised while the fibre-optic system completely ignores the presence of such spurious inputs.

\section{Hydro-dynamic load measurements in a water tunnel using a 2-component fibre optic balance}

\subsection{Experimental setup}

Figure 8 shows a view of the $1 \mathrm{~m} \times 1 \mathrm{~m}$ water tunnel located at the Department of Mechanical Engineering at IISc. The tunnel has a free top surface open to the atmosphere and driven by a

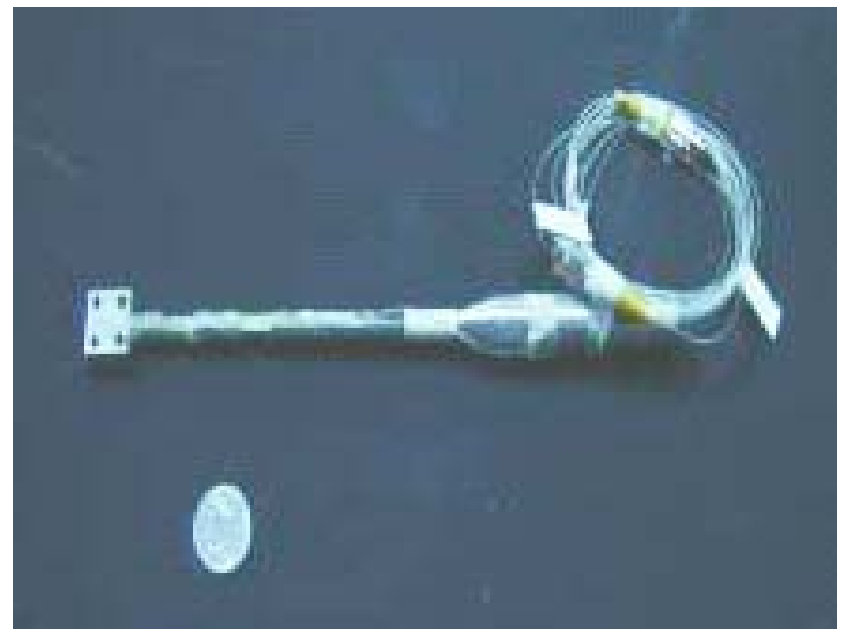

Figure 9. A view of the fibre-optic balance. 


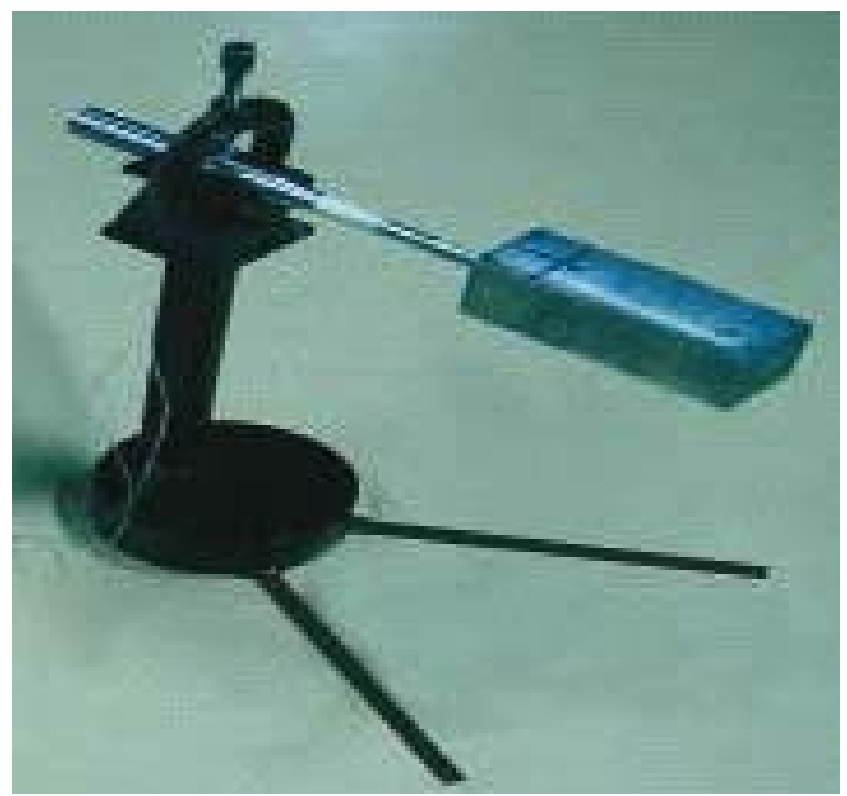

Figure 10. A view of the balance mounted on to the calibration body.

$15 \mathrm{~kW}$ motor with an associated thyristor speed-control system. The maximum water speed was limited for the current experiment to $1.3 \mathrm{~m} / \mathrm{s}$. A calibration of the tunnel was obtained using a pitot-static tube connected to a manometer filled with a fluid with a relative density of 1.62. A colouring agent was used with manometer fluid to easily identify the changing levels during the experiment.

\subsection{Details of the two-component balance and calibration setup}

Figure 9 gives a view of the balance used for the measurements reported in the paper. The instrument is a 4-component balance which is capable of measuring the normal and side

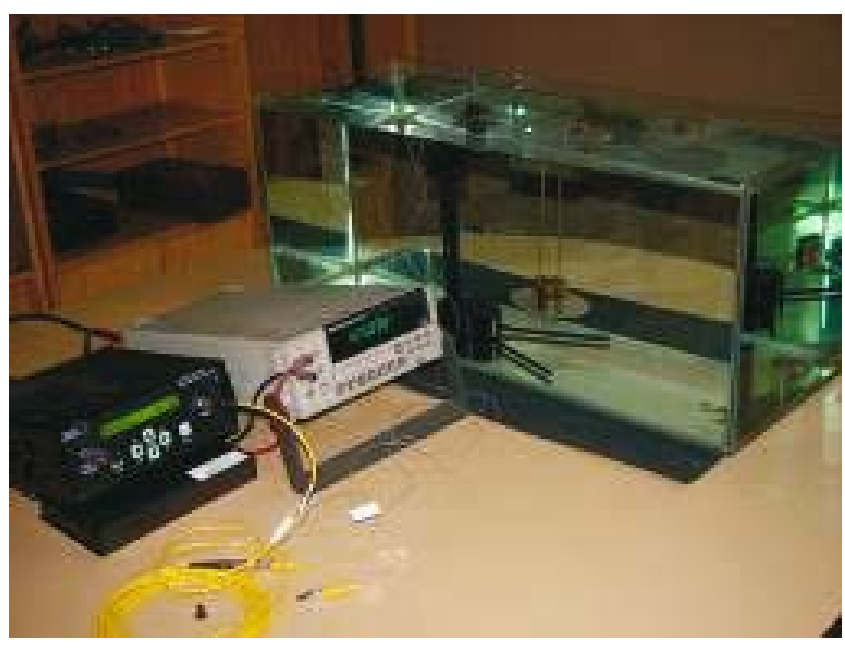

Figure 11. A view of the balance mounted on to the calibration body immersed in water (fish tank). 
forces as well as pitching and yawing moments. However, only two fibre-optic strain gauges were mounted onto the normal force stations $\mathrm{N} 1$ and $\mathrm{N} 2$ which in the present case enable only measurement of normal force and pitching moment. The balance was mounted onto a finely machined calibration body as shown in figure 10. The entire loading setup was placed under water in a fish tank measuring $3 \times 1.5 \times 1.25 \mathrm{ft}$ as shown in figure 11 . Predetermined loads were applied to the balance and the resulting calibration curves are shown in figure $12 \mathrm{a} \& \mathrm{~b}$. The fibre-optic processor was set at an amplifier gain that gave $10 \mathrm{mV}$ output for every microstrain produced on the N1 and N2 flexures in the balance. Loads were applied in steps of 100 grams upto a total weight of 1000 grams at each of the gauge locations.

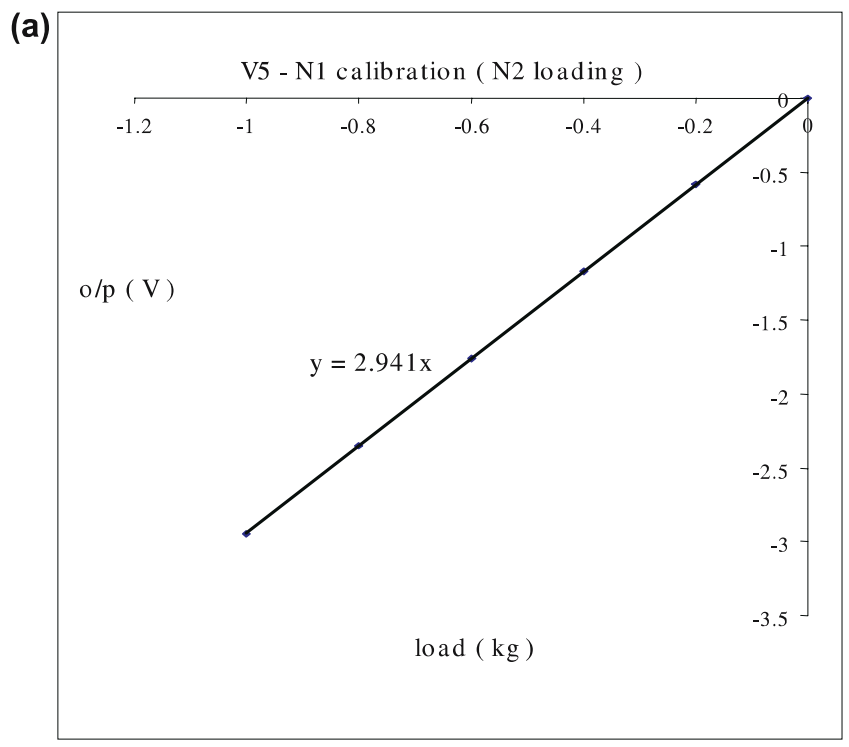

(b)

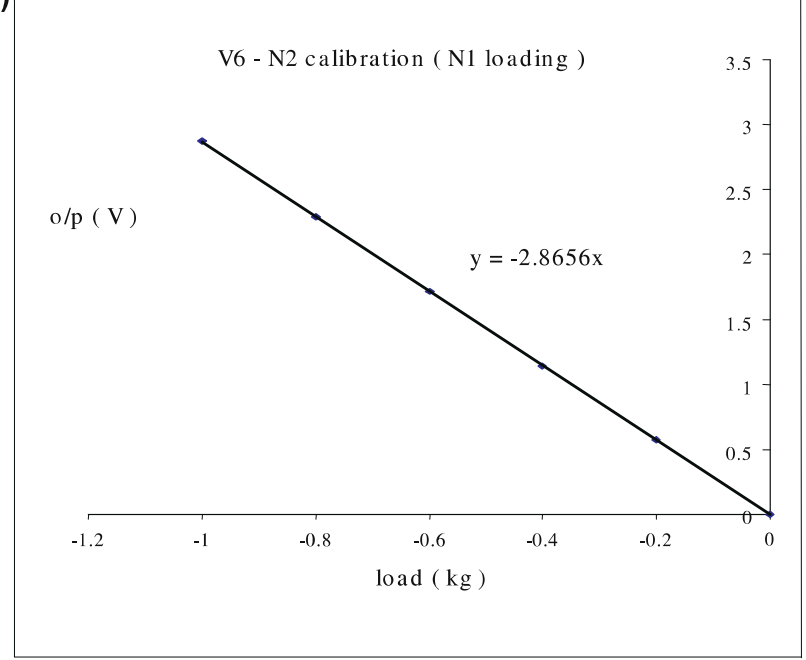

Figure 12. (a) $\mathrm{N} 1$ and (b) N2 calibration curves. 


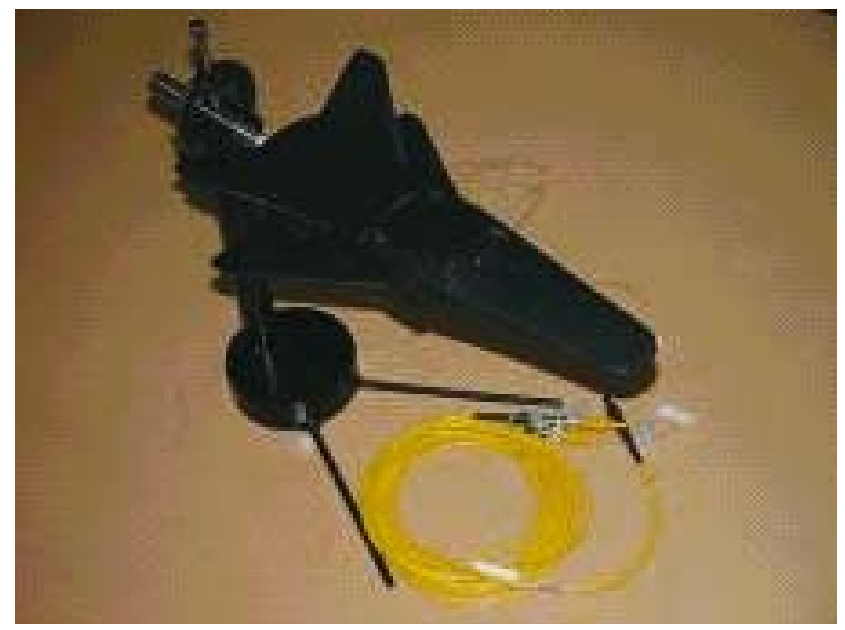

Figure 13. A view of the fibreoptic balance mounted inside the model

\subsection{Models used in the present experiment}

Two models were used for the current measurements. Figure 13 shows the fibre-optic balance mounted inside a generic lifting hypersonic research vehicle model. The 1:20 scale model is made of carbon fibre with associated resins. The model has provision for receiving the internal fibre-optic balance in the form of an embedded steel plate with appropriate threaded holes. A clear gap of $2 \mathrm{~mm}$ is provided separating the model and the balance to ensure proper load measurement even when the model is deflected due to hydrodynamic loads. The other model, a 2-D flat plate was also used but the results of which are not reported here.

\subsection{Results and discussions}

The generic hypersonic research vehicle model was mounted onto a model incidence system in the form of a vertical flat plate as shown in figure 14 . This vertical plate is in turn mounted onto a $T$-section made of steel which runs over the glass side walls of the water tunnel. The

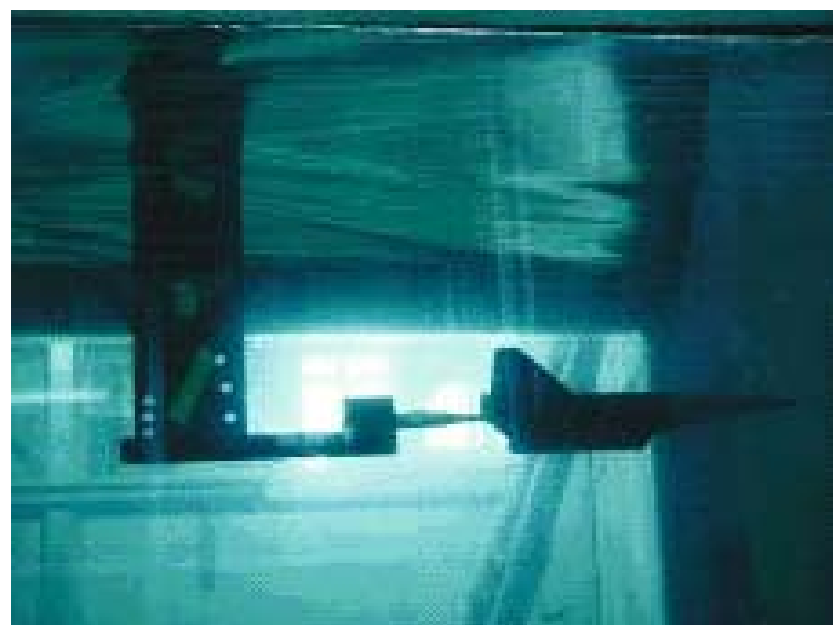

Figure 14. A view of the model incidence system. Angle of attack can be varied from $0^{\circ}$ to $20^{\circ}$ respectively. 
two-channel fibre-optic balance was connected to the signal processor and the outputs of the balance are set to zero before starting the water tunnel. The model incidence system is capable of setting the angle of attack at $0^{\circ}, 10^{\circ}, 20^{\circ}$, and $30^{\circ}$ with an accuracy of $\pm 0 \cdot 2^{\circ}$. The drive RPM was slowly increased from 0 to 1200 in steps of 200. A Pentium IV laptop computer hooked to an Iotech Daqbook data acquisition system with a sixteen-channel A/D converter working at $333 \mathrm{kHz}$ was used for recording the data.

At each tunnel speed, data were recorded for $90 \mathrm{~s}$ with a sampling rate of 500/s. Both N1 and N2 bridge outputs were recorded one after another as the fibre Scan 2000 processor was capable of handling only one channel at a time. Simultaneously, water velocity was recorded using the pitot-static probe connected to the manometer system described earlier. Experiments were carried out at varying angles of attack $\left(0\right.$ to $\left.20^{\circ}\right)$ and at varying tunnel speeds from 0 to $1200 \mathrm{RPM}(0$ to $1.31 \mathrm{~m} / \mathrm{s})$.

Figure 15 gives the output of both $\mathrm{N} 1$ and N2 bridges at varying tunnel speeds and at a fixed angle of attack of $20^{\circ}$. The total normal force at $\alpha=20^{\circ}$ is given in figure 16 . As seen

(a)

N1 output V/S Flow Velocity Alpha $=20 \mathrm{deg}$

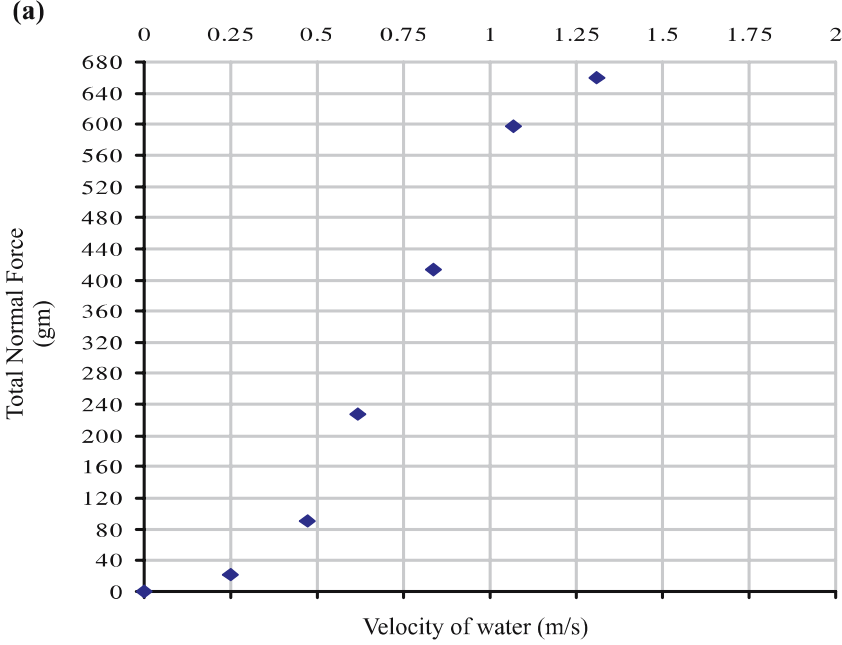

(b)

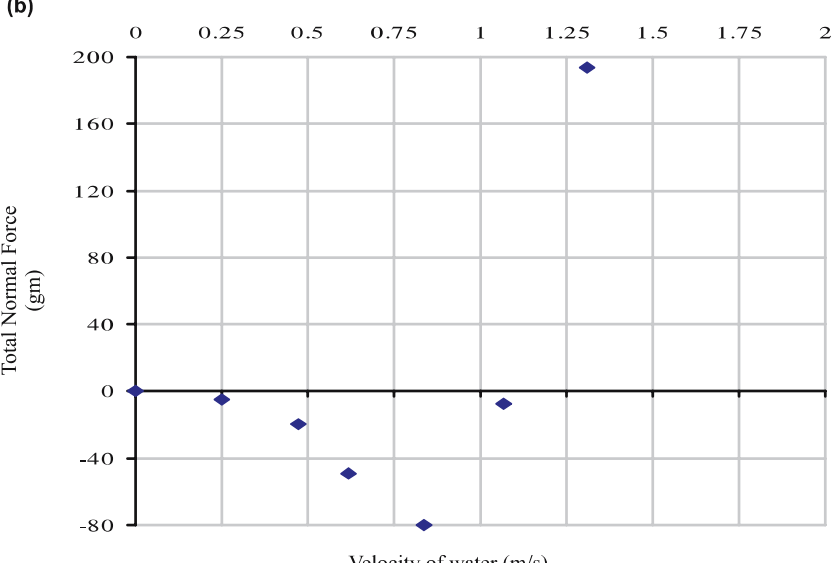

Figure 15. (a) $\mathrm{N} 1$ and (b) $\mathrm{N} 2$

Velocity of water $(\mathrm{m} / \mathrm{s})$ outputs at $\alpha=20^{\circ}$. 


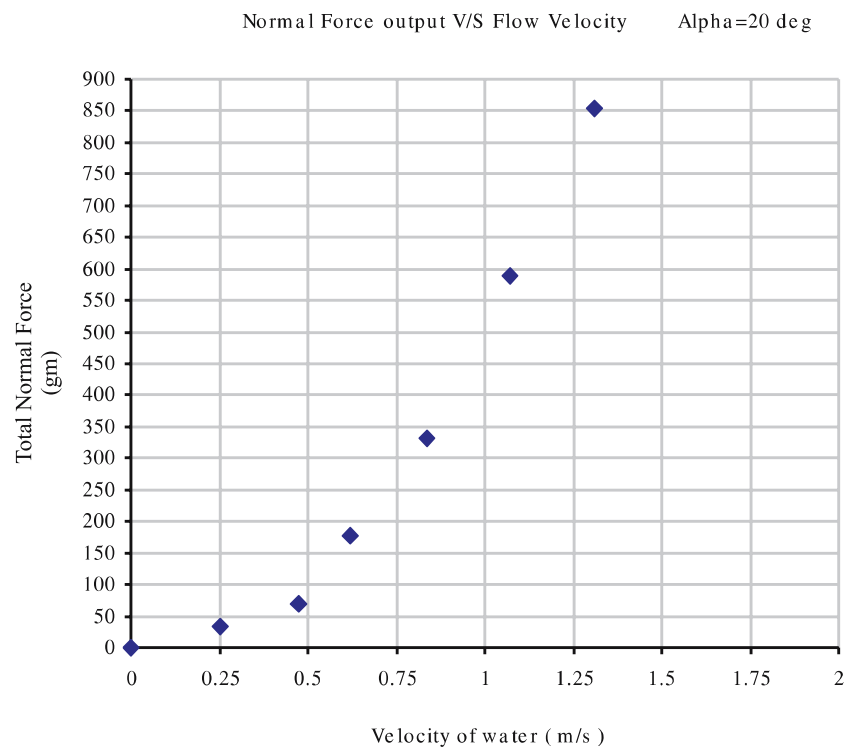

Figure 16. Plot showing the total normal force $(\mathrm{gm}) \mathrm{v} / \mathrm{s}$ velocity of water $(\mathrm{m} / \mathrm{s})$ at $\alpha=20^{\circ}$.

from this figure, the normal forces go up as the tunnel speed is increased. The total normal force (sum of $\mathrm{N} 1$ and $\mathrm{N} 2$ ) as a function of water velocity is plotted in figure 17 for all angles of attack from 0 to $20^{\circ}$. It is found that the fibre-optic balance performed extremely well with a very high level of repeatability and very little hysteresis. It was indeed very gratifying as the fibre-optic balance developed at IISc was indeed the first ever system to be used for load measurement under water, and confirmed by the fibre-optic strain-gauge supplier M/s. Lunavision, USA.

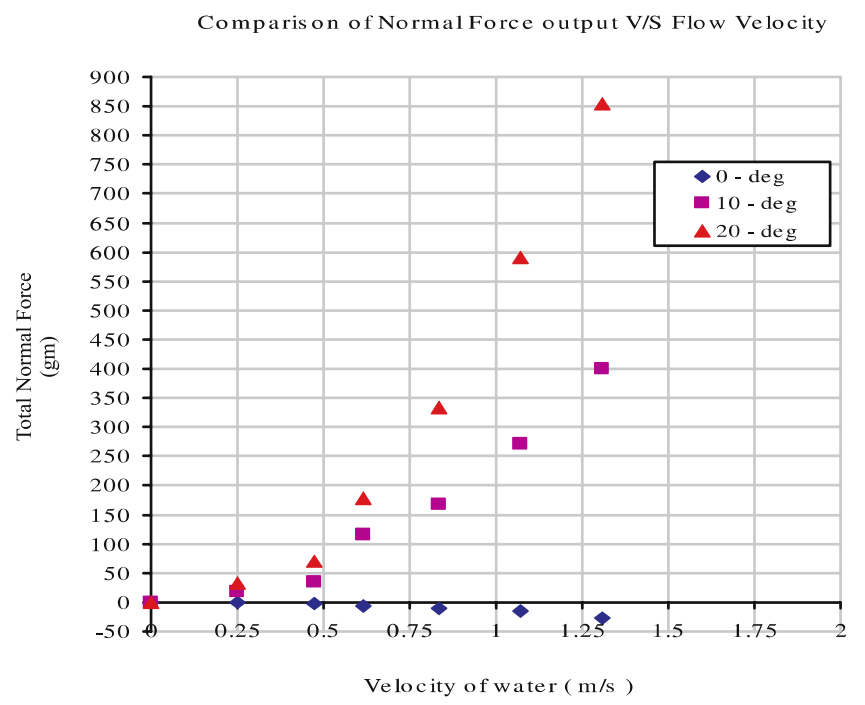

Figure 17. Plot showing the total normal force $(\mathrm{gm}) \mathrm{v} / \mathrm{s}$ velocity of water $(\mathrm{m} / \mathrm{s})$ for angle of attack $0^{\circ}, 10^{\circ}, 20^{\circ}$. 


\section{Conclusions}

It has been a long journey at IISc, since 1998, towards pioneering development of several applications in the area of fluid-dynamic load measurements using fibre-optic sensors. The first-ever multi-component wind tunnel balance using fibre-optic strain gauges was built and used extensively by the IISc group in 1999. Subsequently, extremely small loads of the order of 10 to 30 milligrams of skin friction applied on a floating flat surface at low speeds $(\sim 20 \mathrm{~m} / \mathrm{sec})$ as well as similar loads ( $\sim 100$ to 200 milligrams) at Mach 8.35, were accurately measured using Philtech fibre-optic displacement sensors (Vasudevan et al 2003). Considerable progress was achieved in the measurement of normal force/pitching moment and drag in small balances wherein both conventional (foil-type strain-gauge) as well as EFPI fibre-optic strain gauges were simultaneously used to evaluate the relative performances of the two systems under adverse conditions of flow where the test medium was heated to elevated temperatures upto $800 \mathrm{~K}$ and at Mach numbers of 7.0 and 8.35. A simple and elegant external balance was also built to measure the drag of an automobile model in a large $1 \mathrm{~m} \times 1 \mathrm{~m}$ subsonic wind tunnel enhancing the usefulness of the fibre-optic strain-gauge system (Vasudevan et al 2004). Lastly and most recently, a two-component fibre-optic strain-gauge balance was built and used for the first time in a water tunnel to measure hydro-dynamic loads on various models of interest. To the best of our knowledge, this is the first time that such a simple and elegant fibre-optic balance was used to measure accurately the hydro-dynamic loads under conditions where conventional load measurements are difficult and very expensive. We hope to extend the load measurements at highly elevated temperatures of the order of $1000 \mathrm{~K}$ or more to prove the versatility and unique advantages of using fibre-optics under circumstances where conventional techniques may prove to be totally inadequate.

The authors dedicate this paper for Dr P R Viswanath, a renowned aerodynamicist, on his sixtieth birthday. We thank Profs. Jaywant Arakeri, R Govardhan, and the staff of the Water Tunnel Laboratory at the Department of Mechanical Engineering, IISc for successful conduct of the underwater experiments.

\section{References}

Vasudevan B 2003 Development of a six-component fibre-optic based wind tunnel balance. Annual Progress Report, submitted to AR\&DB, Report 149, Aerodynamics Panel, April

Vasudevan B Hypersonic testing at IISc. NAL-UNI Lecture Series-12, pp. 285-305

Vasudevan B, Jagdeesh H, Padbidri S, Chandra Kishore M, Ravi V, Karale S, Srihari G K, Rudresh C 2005 Aerodynamic load measurement at hypersonic speeds using internally mounted fibre-optic balance system. 21 st Int. Conf. Instrumentation in Aerospace Simulation Facilities. (Sendai, Japan: Sendai Int. Center)

Vasudevan B, Sharan K C, Ashwin K 1998 Fibre optics: Measurement tool in fluid mechanics. Symp. on Adv. in Fluid Mechanics, 2003 (Bangalore: Jawaharlal Nehru Centre for Adv. Sci. Res.) pp 76-85

Vasudevan B, Sharan K C \& Ashwin K C 2004 Fibre optics measurements in wind tunnels. 4th Int. Symp. strain-gauge balance (San Diego, USA) 